\title{
Síntesis social y abstracción idealista. Tentativas materialistas sobre la filosofía del idealismo ${ }^{1}$
}

\author{
Chaxiraxi Escuela $\mathrm{Cruz}^{2}$
}

Recibido: 17 de enero de 2018 / Aceptado: 24 de septiembre de 2018

Resumen. Este artículo presenta una lectura de los momentos de afinidad y diferencia que existen entre las propuestas de interpretación materialista de la filosofía kantiana en A. Sohn-Rethel y Th. W. Adorno. Con sus propios matices, ambos autores utilizan las nociones de fetichismo de la mercancía y síntesis social para mostrar que el concepto de sujeto trascendental no sólo representa el índice de la máxima abstracción que se persigue en la teoría idealista de conocimiento, sino también la forma de la sociedad como sistema funcional de dominio.

Palabras clave: abstracción real, idealismo, materialismo, mercancía, síntesis social, sujeto trascendental.

\section{[en] Social synthesis and idealistic abstraction. Materialistic attempts on the philosophy of idealism}

\begin{abstract}
This paper presents a comparative account of the developments of A. Sohn-Rethel's and Th. W. Adorno's interpretation of Kantian Philosophy from a materialistic position. They utilize the notions of commodity fetishism or social synthesis in order to articulate their theories of social domination. Their analyzes show that the concept of the transcendental subject represents not only something abstract, but the society unaware of itself.
\end{abstract}

Keywords: real abstraction, idealism, materialism, commodity, social synthesis, transcendental subject.

Sumario. 1. Introducción. 2. La irreductibilidad de objeto en Kant. 3. La liquidación materialista del apriorismo en A. Sohn-Rethel. 4. Mercancía y abstracción real. 5. La recepción de las tesis de SohnRethel en Dialéctica Negativa. 6. Intercambio y "memoria de la totalidad". 7. El sujeto como forma de reflexión sobre la falsa objetividad. 8. Conclusiones. Referencias bibliográficas.

Cómo citar: Escuela Cruz, Ch. (2019): Síntesis social y abstracción idealista. Tentativas materialistas sobre la filosofía del idealismo, en Revista Anales del Seminario de Historia de la Filosofía 36 (2), 517-536.

\footnotetext{
1 Este trabajo se inserta dentro del proyecto de investigación: «Justicia, Ciudadanía y Vulnerabilidad. Narrativas de la precariedad y enfoques interseccionales». Referencia: FFI2015-63895-C2-1-R.

2 Universidad de La Laguna. cescuela@ull.edu.es https:/orcid.org/0000-0002-6684-1123
} 


\section{Introducción}

Entre noviembre de 1931 y febrero de 1932, el Institut für Sozialforschung de Frankfurt acogió un seminario de discusión, dirigido por Max Horkheimer, en el que participaron algunos estudiantes avanzados, doctorandos y asistentes, como Theodor W. Adorno, Carl Dreyfus o Leo Löwenthal ${ }^{3}$. Si bien el tema sobre el que giraron las discusiones en este Privatissimum fue la relación entre ciencia y sociedad a la luz de la crisis del idealismo, se planteaba también el interés en elaborar una teoría materialista del conocimiento como alternativa al paradigma idealista. En una de sus múltiples intervenciones, Adorno proponía centrar el debate en lo que a su juicio constituían los momentos fundamentales que ayudaban a diferenciar las tesis materialistas de las idealistas: el problema del sentido y la representación de la figura de la identidad:

"El idealismo se entiende aquí como una visión del mundo en la que los hechos y sus conexiones tienen un sentido, en la medida en que están disponibles o son adecuados para su interpretación. Ellos tienen un significado que es transmitido desde lo real como su símbolo, pero que es reemplazado por este símbolo para volverse independiente" ${ }^{4}$.

El concepto de idealismo que se desprende de estas consideraciones no sólo designa un periodo histórico concreto, sino el impulso de sistematización y de totalidad que acompaña como marca inevitable al pensar ${ }^{5}$. Equivale en sentido general a la absolutización de la identidad entre sujeto y objeto, por medio de la cual se pretende proporcionar integridad y coherencia a lo disperso para hacer desaparecer los componentes cualitativos y diferenciadores del objeto. "En el idealismo se da un dominio del espíritu, allí donde solo ve dos posibilidades. Primero, que el espíritu se presente como lo contrario a la materia o, segundo, que la materia sea por sí misma espíritu, o producto de éste" 6 . Ya desde sus primeros ensayos, Adorno había entendido como el "error fundamental" de la epistemología tradicional la separación entre sujeto cognoscente y objeto conocido. La filosofía moderna instaura la prioridad y el carácter constituyente del sujeto, propiciando la separación entre los polos subjetivo y objetivo a fin de lograr su posterior reconciliación en la identidad con un primero capaz de aprehender de manera inmediata una realidad descualificada y separada. La ruptura con la intentio recta había constituido un paso previo necesario para justificar la posterior afirmación del sujeto como determinador formal de un objeto reducido a datidad abstracta: "Radicalmente separado del objeto, el sujeto lo reduce a sí mismo; devora al objeto en el instante en el que olvida que él mismo es objeto" . De esta articulación de la relación entre el sujeto y el objeto en la teoría del conocimiento

3 Los protocolos de las discusiones llevadas a cabo en este seminario se encuentran recogidos bajo el título Wissenschaft und Krise zwischen Idealismus und Materialismus en: M. Horkheimer, Gesammelte Schriften, vol. 12. Frankfurt a.M.: Fischer, 1985, pp. 349-397.

$4 \quad$ M. Horkheimer, Wissenschaft und Krisis, op. cit., p. 383.

5 Como había confesado en una carta al compositor austriaco Ernst Krenek, "el poder que ejerce el pensamiento idealista sobre nosotros es inmenso, puesto que todas las categorías que usamos de manera ingenua son en verdad idealistas" (Th. W. Adorno; E. Krenek. Briefwechsel. Frankfurt a.M.: Suhrkamp, 1974, p. 87).

$6 \quad$ M. Horkheimer, Wissenschaft und Krisis, op. cit., p. 387.

7 Th. W. Adorno, "Zu Subjekt und Objekt". En: Gesammelte Schriften (en adelante, GS) vol. 10.2. Frankfurt a.M.: Suhrkamp, 1977, p. 742 . 
idealista se desprende una comprensión de la filosofía como sistema de conocimiento capaz de producir el mundo desde sus categorías, y también de captar la totalidad de lo real para identificarla con lo racional. Por eso, la división sujeto-objeto termina por convertirse en una praxis cognoscitiva de dominio pues "tan pronto como la separación se produce sin mediación, se convierte en ideología".

El giro hacia el materialismo en las tesis de Adorno se va dibujando desde sus primeros trabajos como consecuencia del propio cuestionamiento de la actualidad filosófica, tras asumir la crisis del idealismo como un factum histórico-filosófico y plantear la posibilidad de una alternativa al criterio de explicación idealista basado en las ideas de totalidad y sentido. "La crisis del idealismo equivale a una crisis de la pretensión filosófica de totalidad", ", había señalado en su conferencia inaugural de 1931. Por eso, la adopción de un "punto de partida no idealista" ${ }^{10}$ que, sin embargo, conservara la fuerza de la tradición crítica ilustrada, supone tomar consciencia de la quiebra de la forma tradicional de fundamentación teórica. Abandonar la "ilusión" de aprehender la totalidad de lo real con la fuerza del pensamiento, exigía al mismo tiempo revocar la reducción al sujeto que había primado en el curso de la reflexión epistemológica moderna. Una cuestión que ve posible en la defensa de una afinidad material entre sujeto y objeto de conocimiento, y en el desarrollo de un ejercicio de liquidación inmanente del idealismo, es decir, una crítica al principio de identidad y de sistema, para conducirlos al instante en el que sus antinomias y aporías se muestren con mayor claridad. Ve concretar esta tarea en dos cuestiones específicas a las que debe hacer frente la metacrítica materialista de la teoría del conocimiento ${ }^{11}$. En primer lugar, en el problema de la relación entre concepto y cosa, cuya identidad entiende como el "nervio vital" del pensamiento idealista. En segundo lugar, en la cuestión de la hipóstasis del espíritu en la teoría del conocimiento, esto es, la figura de la subjetividad trascendental. Por eso, su posición ante la filosofía del idealismo alemán adopta como punto de partida la crítica a la teoría de la abstracción. Síntesis y sujeto se convierten entonces en los principales conceptos en los que anclar la interpretación del idealismo, pues expresan "un contenido de verdad que se muestra en la preformación del concepto de sujeto a partir de una forma específica de la objetividad social"12.

La dialéctica negativa busca desenmascarar la proclamada autonomía del sujeto y desvelar los límites de la imagen idealista de una subjetividad dotadora de sentido, así como su papel fundante en la teoría del conocimiento. Adorno se refiere a las filosofías de Kant y Husserl como máximos exponentes del esquema idealista de la unidad de conciencia. El yo trascendental representa la máxima expresión de la abstracción y del alejamiento del ente empírico que persigue la epistemología moderna, al proponer la fundamentación de las leyes del pensamiento en la síntesis trascendental. Sin embargo,

\section{Ibid.}

9 Th. W. Adorno, "Die Aktualität der Philosophie”. En: GS, vol. 1. Frankfurt a.M.: Suhrkamp, p. 326. Demirovic entiende la intención de estos trabajos tempranos como el esfuerzo por "presentar un concepto de dialéctica que, de manera distinta a la hegeliana, fuerce la identidad de concepto y objeto, para alcanzar a través del sistema un antisistema y un pensamiento concreto" (A. Demirovic, Der nonkonformistische Intelektuelle. Die Entwicklung der Kritischen Theorie zur Frankfurter Schule. Frankfurt a.M.: Suhrkamp, 1999, p. 632).

10 M. Horkheimer, Wissenschaft und Krisis, op. cit., p. 391.

11 Cfr. Th. W. Adorno. Vorlesung über Negative Dialektik. 1965-66. Frankfurt a.M./Berlín: Suhrkamp, 2003 , p. 37.

12 C. Thein. Subjekt und Synthesis. Eine kritische Studie zum Idealismus und seiner Rezeption bei Adorno, Habermas und Brandom. Würzburg, Königshausen \& Neumann, 2013, p. 335. Thein insiste en ver la crítica a la teoría de la abstracción como el caballo de batalla de Adorno ante la filosofía del idealismo alemán. 
a diferencia de la fenomenología, su lectura de Kant le ofrece la posibilidad de formular una dialéctica materialista que, por un lado, comprenda el concepto de subjetividad transcendental como lugar en el que se fundan las condiciones de posibilidad del conocimiento objetivo. Pero también, por otro, que se desprenda del carácter dogmático de retirada a la objetividad. Su interés se dirige, pues, a la necesidad de retomar la teoría del conocimiento de Kant desde una perspectiva crítico-materialista.

\section{La irreductibilidad del objeto en Kant}

Pese a que Adorno no dedicó ningún monográfico a interpretar la obra de Kant, ésta ocupa un lugar destacado en sus textos desde que, siendo adolescente, tuvo un primer acercamiento de la mano de su amigo y mentor Siegfried Kracauer. Kant también es uno de los ejes centrales de las lecciones académicas que impartió en Frankfurt tras los años de exilio, como las que dedicó en la segunda mitad de los años cincuenta y principios de los sesenta a la Crítica de la razón pura y la Crítica de la razón práctica respectivamente ${ }^{13}$. En ellas es posible encontrar una interesante introducción a Kant que lejos de centrarse en el estudio de los tecnicismos terminológicos o en la lectura de los pasajes canónicos de su obra, presta atención a momentos por los que la historiografía filosófica ha pasado casi de puntillas. Como explicaba a los estudiantes que llenaban sus aulas, "quería traducir la obra kantiana desde una imagen rígida y codificada a otra abierta. Invitarles a concebir esta filosofía como un campo de fuerzas donde, detrás de conceptos muy abstractos que entran en conflicto unos con otros $(. .$.$) hay en realidad fuerzas de una experiencia extraordinariamente viva"14.$

En estos cursos, Adorno insiste en presentar el interés de la teoría kantiana no en una vuelta al sujeto, sino a la objetividad de la experiencia. El giro copernicano de la filosofía trascendental no convierte al sujeto en la medida de lo que a partir de entonces se deba reconocer como lo real. Por el contrario, ve en él elementos que permiten interpretar la de Kant como una filosofía del objeto. Pues ni la objetividad se fundamenta de forma ontológica, ni la subjetividad se concibe como absoluta. Esto significa adoptar como máxima epistemológica la conciencia de la irreductibilidad del sujeto y del objeto en el conocimiento:

«Podemos decir que el pensamiento dialéctico está ya contenido objetivamente en la teoría kantiana incluso aunque el propio Kant lo mantuviera a la sombra de un dualismo entre forma y contenido (...) Esos dos conceptos están cada uno de ellos mediados por el otro. Pueden ver aquí, por tanto, que la dialéctica no implica brujería alguna, sino más bien que la transición a la dialéctica es una consecuencia necesaria que se desprende de la figura objetiva de la filosofía de Kant» ${ }^{15}$.

13 A comienzos de 2014 y bajo la dirección de Axel Honneth y Dirk Braunstein se puso en marcha el proyecto editorial "Die Frankfurter Seminare Theodor W. Adornos. Edition und Publikation der Gesammelten Sitzungsprotokolle", con el que se pretende la publicación de los protocolos completos de las lecciones impartidas por Adorno desde 1949 hasta 1969. Cfr. D. Braunstein. "Das Denken der Kritischen Theorie. Die Sitzungsprotokolle aus den Seminaren Theodor W. Adornos". En: M. Endreß, et al. Zyklos 1. Jahrbuch für Theorie und Geschichte der Soziologie. Wiesbaden: Springer VS, 2014, pp. 283-309.

14 Th. W. Adorno. Kants Kritik der reinen Vernunft. Frankfurt a.M.: Suhrkamp, 1995, p. 13. Adorno imparte este curso en el semestre de verano de 1959.

15 Th. W. Adorno. Kants Kritik, op. cit., p. 91. 
Lo que pretende rescatar Adorno será, precisamente, este momento de «irreductibilidad» que permite comprender la filosofía kantiana como una teoría de la objetividad. La atención sobre la recuperación de la dignidad de la experiencia como organon del pensamiento, que pronto se convirtió en un momento central en su proyecto materialista, tiene como antecedente la filosofía kantiana, pues en ella el conocimiento de la experiencia no parte de una percepción subjetiva, sino que aspira a la construcción necesaria y objetiva de la universalidad. Aquí sitúa además el momento dialéctico que encuentra en Kant, así como también las aporías que constituyen la clave de su lectura. De un lado, prevalece una pretensión de sistema, pues la razón sólo puede ser pensada como una unidad cerrada en sí misma. De otro, se desarrolla la conciencia del "bloque" o límite, esto es, la existencia de lo heterogéneo irreductible a ella misma. Esto la distingue tanto de los desarrollos posteriores del idealismo en los que desaparecen los anclajes objetivos de la razón, como también del positivismo, pues si bien coincide con éste en la conciencia de la finitud del conocimiento, en Kant prevalece un sentimiento de "tristeza metafísica" cuerdo de aquello que no puede ser reducido ni al sujeto, ni a lo dado:

"La subjetivización tal como la efectúa la filosofía de Kant frente al realismo ingenuo, es decir, frente a la creencia ingenua en la realidad de la cosa en sí, ante la que me encuentro como sujeto pensante y sensible, esta subjetivización, como la defendieron Kant y Fichte, significa una cosificación en menor medida; en cierto modo, la filosofía kantiana entiende el mundo mucho más como un proceso, y mucho menos como algo cuajado y cosificado"17.

En este campo de fuerzas, la figura del sujeto trascendental constituye un momento central. La Dialéctica negativa asume la tarea de desmontar la falacia de la subjetividad constitutiva como ideal de la filosofía moderna, lo que supone enfrentarse a lo que Adorno considera la cuestión central que articula la Crítica de la razón pura: "El que la razón prescriba las leyes a la naturaleza, no significa más que la subsunción bajo la unidad de la razón. Ella proyecta esa unidad - es decir, su propio principio de identidad - a los objetos y luego la hace pasar por el conocimiento que tiene de ellos"18. Lo que busca la dialéctica adorniana es descifrar, con ayuda de la propia fuerza del sujeto, la objetividad que permanece oculta en las condiciones constitutivas de la subjetividad. Las estructuras aparentemente inmutables de la subjetividad trascendental tienen su origen en los procesos de dominación que se dan en la sociedad, de ahí que oculten una abstracción ejercida a partir de subjetividades históricamente devenidas. Por eso, señala Adorno, "el formalismo aparentemente extremo de la filosofía kantiana incluye en sí mismos momentos de cambios repentinos hacia lo material"19. El contenido oculto de la síntesis trascendental es, como se verá a continuación, el trabajo social y el principio de intercambio, un contenido cuyo descubrimiento atribuye principalmente a la obra de Alfred Sohn-Rethel, a quien reconoce como el primero en haber comprendido el entrelazamiento entre razón y realidad social que se encuentra almacenado en los presupuestos de la teoría idealista del conocimiento.

\footnotetext{
Th. W. Adorno. Kants Kritik, op. cit., p. 268.

Th. W. Adorno. Kants Kritik, op. cit., p. 173.

Th. W. Adorno, Negative Dialektik. En: GS, vol. 6, Frankfurt a.M.: Suhrkamp, 1970, p p. 254.

Ibid., p. 263.
} 


\section{La liquidación materialista del apriorismo en A. Sohn-Rethel}

La obra de Sohn-Rethel representa uno de los abordajes teóricos más originales, y quizás también más desatendidos, de Marx. Cercano a los planteamientos de los teóricos críticos de Frankfurt, nunca tuvo una vinculación formal con el Instituto, una ausencia de soporte institucional que puede explicar la desatención que sufrió su obra durante varias décadas. Sohn-Rethel nace en París en 1899, aunque pronto se traslada a Heidelberg donde asiste a clases de economía, filosofía y sociología impartidas por profesores como Ernst Cassirer, Emil Lederer o Max Weber. Se doctora en 1928 con una tesis sobre la crítica a la economía política en Schumpeter. ${ }^{20}$. Durante sus años como estudiante en Berlín, Darmstadt y Heidelberg había tenido sus primeros contactos con la lectura de textos marxistas, y ya entonces comenzaba a fraguarse su convencimiento de la necesidad oponer una explicación materialista a la teoría idealista del conocimiento.

El ascenso del nacionalsocialismo a partir de 1932 lo obligó a exiliarse en Suiza. Será en estos años cuando retome sus investigaciones y escriba su primer trabajo, el llamado Exposé zum Plan einer soziologischen Theorie der Erkenntnis ${ }^{21}$, cuyas copias hizo llegar a Benjamin, Bloch, Adorno, Horkheimer y Lukács, con la esperanza de recibir financiación del Institut. Precisamente bajo la influencia de Adorno y de Benjamin, Sohn-Rethel escribe en 1937 Kritische Liquidierung des Apriorismus, ensayo en el que desarrolla las bases de un programa materialista de conocimiento que posteriormente se concretaría en sus dos obras más importantes, Geistige und körperliche Arbeit y Warenform und Denkform. El trabajo, que se interroga sobre el vínculo entre las epistemologías modernas y el desarrollo de la producción y el intercambio de mercancías en la sociedad capitalista, se marca como objetivo proponer una teoría materialista del conocimiento y de lo social capaz de entrelazar la epistemología kantiana con la crítica a la economía política de Marx. Se opone tanto al neokantismo como al marxismo esclerotizado, para enunciar un propósito fundamentalmente práctico: la transformación del ser social. Lo que subyace a este objetivo es el interés por desarrollar una "explicación genética" del conocimiento que lograra oponer "la explicación materialista del pensamiento racional a su interpretación idealista, de manera que quedara resuelta la fetichización de la ratio"22. Esclarecer la génesis del conocimiento significa, pues, mostrar sus condiciones materiales de validez, con lo que se probaría que la verdad es históricamente condicionada y no atemporalmente absoluta. Sohn-Rethel insistía en determinar la esencia del método materialista de conocimiento como la exigencia de no emplear ninguna categoría de la cual no se sepa por qué relaciones de producción es condicionada. Por eso, el análisis crítico del concepto de cosificación que realiza en el Exposé se convierte en el punto de partida indispensable para la investigación histórico-materialista, puesto que no solo ayuda a corregir la apariencia de validez atemporal de las categorías lógicas del pensamiento, sino también el carácter de pseudonaturalidad de lo históricamente constituido.

20 Cfr. A. Sohn-Rethel. Von der Analytik des Wirtschaftens zur Theorie der Volkswirtschaft. Frühe Schriften. Freiburg i.B.: Ça-ira Verlag, 2012.

21 Cfr. A. Sohn-Rethel. Soziologische Theorie der Erkenntnis. Frankfurt a. M.: Suhrkamp, 1985.

22 A. Sohn-Rethel. "Kritische Liquidierung des Apriorismus. Eine materialistische Untersuchung". En: Geistige und körperliche Arbeit. Zur Epistemologie der abendländlische Geschichte. Weinheim: VCH, Acta Humaniora, 1989, p. 154. 
Pese a la malograda financiación de su trabajo, el texto se convirtió rápidamente en objeto de discusión con Adorno, iniciándose una interesante correspondencia entre ambos que duraría más de una década. En una misiva de 1936 conocida como "Carta de Nottingham"23, Sohn-Rethel explica al frankurtiano su interés en llevar adelante dos intenciones que habían determinado sus trabajos tempranos durante largo tiempo. La primera de ellas era mostrar de qué manera el origen histórico del conocimiento puede ser explicado en cualquier comprensión idealista a partir de una ruptura profunda con la praxis del ser social. La actividad del pensamiento se encuentra históricamente constituida y socialmente condicionada, lo cual conduce a la segunda intención del Exposé: la cuestión de la relación entre el problema de la validez en la teoría del conocimiento y la transformación práctica del ser material. Esto exigía partir de la indicación marxista fundamental de que todos los problemas de la teoría humana se enredan finalmente con los problemas en la praxis:

"El estudio marxista de la mercancía al comienzo de El Capital analiza la economía política, pero no se pregunta por la posibilidad de una síntesis social en las sociedades basada en el principio de la propiedad privada. Por el contrario, mis estudios se centran en la investigación del nexo social. A través de este cambio en el tema, la cuestión político-económica se vuelve sociológica"24.

Sohn-Rethel pretende "corregir" lo que considera una deficiencia fundamental en el materialismo histórico de Marx: la escasa de atención prestada sobre las formas conceptuales de las ciencias naturales como parte de la "superestructura intelectual". En la lectura de Marx se tiene la impresión de que todo lo que constituye la realidad material está incluido en el análisis de las fuerzas productivas y, sin embargo, descuida el papel representado por las ciencias de la naturaleza. Esto ha derivado en una contradicción en la que han caído las posteriores interpretaciones de los textos marxistas, tal como señala en la introducción a la versión inglesa de Trabajo espiritual y trabajo corporal:

"Por un lado, todos los fenómenos del mundo de la conciencia - pasados, presentes y futuros - se interpretan históricamente y se valoran directamente desde un punto de vista temporal. Por otro, los problemas de la lógica, la matemática y la ciencia se interpretan en función de normas de carácter atemporal. ¿Acaso un marxista debe ser materialista en lo relativo a la verdad histórica e idealista en lo que se refiere a la verdad natural?"25.

El problema de la cognición, que en su opinión había sido descuidado por el materialismo histórico, debía ser recuperado desde una perspectiva crítica y materialista. Por este motivo, Sohn-Rethel asume la problemática de la teoría marxiana de la mercancía, pues considera que su desciframiento permite explicar el carácter universal de la economía capitalista y, con ello, también la raíz ideológica de la sociedad.

23 Cfr. A. Sohn-Rethel."Exposé zur Theorie der funktionalen Vergesellschaftung. Ein Brief an Theodor Adorno". En: Geistige und körperliche Arbeit, op. cit., pp. 131-153.

24 A. Sohn-Rethel, Geistige und körperliche Arbeit, op. cit., p. VII.

25 A. Sohn-Rethel. Intellectual and Manual Labour. New Jersey: Humanities Press, 1978, pp. 2-3. La versión en inglés del trabajo, a manos de su hijo Martin, no es una simple transposición del texto alemán, sino que incluye importantes aportaciones, como la introducción a la cual nos referimos en este momento. 
Este objetivo de elaborar un análisis crítico de la teoría idealista del conocimiento a partir de la búsqueda de sus condiciones de posibilidad en la materialidad de las relaciones sociales, encuentra apoyo en algunos de los textos de Marx:

"La crítica marxista de la economía política y nuestra propia crítica de la epistemología burguesa tiene en común un mismo fundamento metodológico: el análisis de las mercancías que se lleva a cabo en los capítulos iniciales de El Capital y, todavía antes, en la Contribución a la crítica de la economía política de 1859. Lo más destacado de esta cuestión es la identidad formal de ambas críticas”26.

Sin embargo, no sólo ve en su análisis la clave para descifrar el carácter universal de la economía capitalista, sino también una explicación histórica y material de las propias formas de pensamiento. Por eso, en lugar de concentrar su análisis en el aspecto formal del valor para priorizar el componente económico del trabajo, SohnRethel pretende llegar hasta la fuente de la que proviene la abstracción, lo cual significa atender a la estructura formal del intercambio de mercancías. La forma de las mercancías y del pensamiento conceptual tendrán su lugar de correspondencia en el proceso práctico del intercambio de equivalentes que se da en la producción capitalista, a través de lo que Sohn-Rethel llamará la "síntesis social". Por eso, se trata de mostrar de qué manera los elementos constitutivos de la abstracción que tiene lugar en el intercambio revelan una inequívoca similitud con los elementos conceptuales del conocimiento 27 .

\section{Mercancía y abstracción real}

La forma mercancía y la ley del intercambio se presentan en el sistema capitalista como el a priori de la producción. La existencia de las mercancías depende de la producción y, al mismo tiempo, las condiciones de posibilidad de la producción son las leyes que hacen posible la existencia de las mercancías en la sociedad: "la existencia inmediata de las mercancías se vuelve su existencia inmediata según leyes, y la existencia inmediata de las mercancías aparece como la consistencia total de la sociedad" ${ }^{28}$. Sin embargo, lo característico del orden social de producción capitalista

26 A. Sohn-Rethel. Intellectual and Manual Labour, op. cit., p. 8. En el prólogo a la Contribución a la crítica de la economía política Marx lleva a cabo una reflexión sobre el método de la Economía Política en torno a dos aspectos fundamentales: la elaboración teórica de la economía y el papel representado por las categorías económicas en los distintos grados de desarrollo de la vida humana: "Como ocurre en cualquier ciencia social histórica, cuando se observa el desarrollo de las categorías económicas es necesario tener en cuenta que el sujeto -aquí la moderna sociedad burguesa - es algo dado tanto en la realidad como en la mente y que las categorías expresan formas de ser, determinaciones de la existencia, a menudo solamente aspectos aislados de esta sociedad determinada, de este sujeto" (K. Marx. "Einleitung zu den Grundrissen der Kritik der politischen Ökonomie”. En: K. Marx; F. Engels. Werke, v. 13. Berlín: Dietz, 1969, p. 637).

27 La presencia de una teoría del conocimiento en la obra de Sohn-Rethel no ha estado ausente de controversia. Wohlrapp, por ejemplo, niega cualquier posibilidad de hablar de una epistemología en la obra del marxista, sino un ejemplo de "las trampas en las que uno cae cuando descuida los requisitos elementales del rigor", unas tesis que el propio autor se encargaría de matizar años más tarde ( $C f r$. H. Wohlrapp. "Materialistische Erkenntniskritik? Kritik an Alfred Sohn-Rethels Ableitung des abstrakten Denkens und Erörterung einiger grundlegender Gesichtspunkte für eine mögliche materialistische Erkenntnistheorie”. En: J. Mittelstraß (ed.) Methodologische Probleme einer normativkritischen Gesellschaftstheorie. Frankfurt a.M.: Suhrkamp, 1975).

28 A. Sohn-Rethel, "Kritische Liquidierung des Apriorismus", op. cit., p. 159. 
es que no se realiza siguiendo una dirección o cooperación planteada de antemano, sino por medio de acciones individuales, independientes las unas de las otras, de personas autónomas. Se trata de un orden completamente funcional, asegura SohnRethel: "sólo la ley funcional del intercambio de las mercancías decide aquí también sobre la realidad objetiva del valor de uso y sobre la validez social del valor de las mercancías" 29 . Por eso, no entiende la idea marxista de "fetichismo" como el análisis de las formas fantasmagóricas que asume la mercancía, sino que presta atención a las bases objetivas propias de la sociedad en la que se manifiesta ${ }^{30}$. Lo que descubre en la estructura formal de la mercancía es la existencia de un proceso de abstracción que funciona como "síntesis social", esto es, una universalización de la abstracción mercantil que convierte en conmensurables e idénticos a individuos y acciones que no lo son. Esta síntesis social no se produce por el proceso de trabajo, sino por el del intercambio. Por eso, si bien no niega la abstracción en el proceso de trabajo capitalista, tampoco la entiende el como origen de la síntesis social. Es la mercancía la que, al imponerse universalmente, subordina al proceso de trabajo y lo convierte en abstracto. Sohn-Rethel niega que la abstracción surja con exclusividad en el modo de producción capitalista, si bien con el dinero entendido como un universal intercambiable logra alcanzar un mayor grado de intensidad.

Esta abstracción no es una propiedad natural de las cosas, sino "una ficción que se vuelve objetiva en la media que la sociedad la requiere independientemente de la voluntad del individuo"31. Lo característico de la abstracción es que no encierra un significado metafórico, sino que tienen un origen social, pues no tiene su origen en las mentes de los hombres, sino que brota de sus acciones concretas, aun si estos ser conscientes de sus consecuencias: "Es abstracción en un sentido preciso y literal. El concepto económico de valor que resulta de ella se caracteriza por una ausencia total de calidad, una diferenciación puramente cuantitativa y aplicable a todo tipo de producto y servicio del mercado" ${ }^{32}$. Sohn-Rethel ve en estas cualidades de la abstracción del valor cierta similitud con las categorías fundamentales de la ciencia natural. Sin embargo, mientras que sus conceptos son abstracciones de pensamiento, el concepto económico de valor es real, por lo que "no existe en ninguna otra parte que no sea en la mente humana, pero no surge de ella. Más bien es de carácter puramente social, pues surge en la esfera espacio-temporal de las interrelaciones humanas. No son las personas quienes originan estas abstracciones, sino sus acciones" ${ }^{33}$. Lo abstracto no es la consciencia de los que intercambian, sino la acción misma del intercambio, asegura, por lo que "nada parece incomprensible para ellos, sus acciones las realizan de mutua acuerdo y en be-

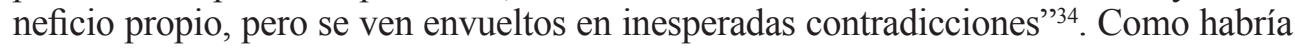
apuntado Marx en El Capital, no lo saben, pero lo hacen.

\footnotetext{
29 Ibid., p. 159.

30 Para un acercamiento al concepto de fetiche en Marx, véase la reciente monografía de C. Ramas San Miguel. Fetiche y mistificación capitalistas. La crítica de la economía política de Marx. Tres Cantos: Siglo XXI, 2018.

31 T. Reichardt. "Aporien der soziologischen Erkenntnistheorie Alfred Sohn-Rethel”. Wissenschaftliche Mitteilungen, 6, 2008, p. 250.

32 A. Sohn-Rethel. Geistige und körperliche Arbeit, op. cit., p. 12.

33 Ibid., p. 13. Sobre esto, Woesler apunta: "El pensamiento abstracto sólo puede explicarse a partir de la segunda naturaleza del hombre, su naturaleza netamente social; guarda una separación estricta de la comprensión primaria de la naturaleza, que se constituye en el trabajo manual y en la producción" (C. Woesler. Für eine begreifende Praxis in der Natur. Geldförmige Naturerkenntnis und kybernetische Natur. Giessen: Focus-Verlag, 1978, p. 97).

34 A. Sohn-Rethel. Geistige und körperliche Arbeit, op. cit, p. 54.
} 
Sohn-Rethel había explicado a Adorno su intención teórica central: "conducir los conceptos de subjetividad, identidad, existencia, cosa, objetividad y lógica de las formas de juicio, a momentos de la forma mercantil de los productos del trabajo, a su génesis y dialéctica" ${ }^{35}$. Al priorizar la abstracción real, rechaza que la relación existente entre los elementos formales de la síntesis social y los del conocimiento obedezca a una mera analogía. Por el contrario, las bases conceptuales del conocimiento se encuentran históricamente condicionadas por la estructura básica de la síntesis social de cada época. Las condiciones de posibilidad del conocimiento son históricas y no apriorísticas, pues no radican en la interioridad de la conciencia, sino que son determinaciones de la abstracción de la síntesis social. "El punto central de nuestro tema", apuntaba, "es la afirmación de que la identidad es un atributo formal históricamente condicionado de las mercancías y una forma de vínculo social entre las personas" ${ }^{36}$. Se trata de explicar de qué manera las principales estructuras de pensamiento de una época determinada se encuentran en estrecha relación con la forma de la síntesis social ahí generada. "La conceptualización filosófica del pensamiento racional está enraizada formal e históricamente en la abstracción real de la síntesis social por medio del intercambio de mercancías, es decir, en la segunda naturaleza" ${ }^{37}$. Mostrar la "secreta, pero firme identidad" 38 entre la forma-mercancía y la forma-pensamiento, conduce al desarrollo de una interpretación materialista de la teoría idealista del conocimiento que presenta el origen de las formas del pensamiento, no como el resultado de un acto espontáneo de la conciencia, sino de los procesos de abstracción que tienen lugar en la sociedad del intercambio. Al mostrar que la abstracción ocurre realmente dentro de la objetividad social, Marx habría señalado la génesis objetiva del pensamiento abstracto del sujeto. Por eso, a diferencia del idealismo trascendental que postula una conciencia que constituye un mundo por medio de sus propias categorías de representación, el análisis del fetichismo de la mercancía trae como resultado una forma de subjetividad histórica determinada como parte del mundo social de la objetividad.

Aquí se sitúa la apropiación que hace Sohn-Rethel de la teoría del conocimiento kantiana. Y es que si bien alaba la consideración que realiza Kant de los componentes básicos de nuestra forma de pensar como preformados, no acepta que esta preformación pueda explicarse desde las condiciones ahistóricas de la razón, o desde una "fantasmagórica síntesis trascendental", sino sólo desde las condiciones materiales y concretas:

"Las categorías son históricas por su origen y sociales por naturaleza, pues llevan a cabo la síntesis social sobre la base de la producción capitalista de mercancías, de manera que la facultad cognoscitiva que articulan es una capacidad social a priori de la mente, aunque su apariencia sea la contraria, la de que obedecen al principio del ego cogito" 39 .

\footnotetext{
A. Sohn-Rethel. "Exposé zur Theorie der funktionalen Vergesellschaftung", op. cit, p. 137.

A. Sohn-Rethel. "Kritische Liquidierung des Apriorismus", op. cit, p. 169.

A. Sohn-Rethel. Geistige und körperliche Arbeit, op. cit, p. 60.

38 A. Kordela. "Materialist Epistemology: Sohn-Rethel with Marx and Spinoza". History of the Human Sciences, vol. 29(2), 2016, p. 116.

39 A. Sohn-Rethel. Intellectual and Manual Labour, op. cit, p. 7.
} 
Por eso, ve en el sujeto trascendental kantiano un elemento esencial de la abstracción del intercambio, pues supone la forma más perfecta de la intercambiabilidad de mercancías y de la síntesis social:

"La unidad trascendental de la autoconciencia, para utilizar una expresión kantiana que describe el fenómeno aquí considerado, es a su vez el reflejo intelectual del elemento más esencial de la abstracción del intercambio: la forma de intercambiabilidad de la mercancías subyacentes en la unidad del dinero y de la síntesis social"40.

La idea que defendería inicialmente en el Exposé de Lucerna, y que continuaría desarrollándose a lo largo de su obra, es que en el interior de la estructura formal de la mercancía se encuentra el sujeto trascendental del conocimiento. Esto suponía situar al sujeto trascendental en el centro mismo de la estructura de la mercancía y mostrarlo como el concepto fetiche de la función capital del dinero ${ }^{41}$. El convencimiento que se estaba fraguando desde sus años de estudiante había logrado concretarse, tal como aseguraría décadas más tarde en una entrevista: "cómo marxista me había quedado claro una cosa mientras no se le diera una respuesta materialista a la pregunta por el sujeto trascendental, el materialismo permanecería injustificado" ${ }^{42}$.

\section{La recepción de las tesis de Sohn-Rethel en Dialéctica negativa}

Las controvertidas tesis de Sohn-Rethel sobre el yo trascendental y el análisis de la mercancía lo alejó del foco de atención en las corrientes dominantes del marxismo, algo de lo que el propio autor tomó consciencia.

"Tan solo algunos espíritus solitarios, outsiders como yo, han tenido intuiciones parecidas, y ninguno de ellos de manera tan empática como Adorno, en cuyo pensamiento, si bien a su manera, se puede encontrar las huellas de la misma verdad. En 1936 estábamos completamente de acuerdo en esta cuestión, si bien luego se ocuparía de cosas muy distintas del análisis de mercancías y de la economía”43.

Adorno y Sohn-Rethel se habían conocido a principios de los años veinte durante una estancia en Capri y posteriormente volverían a encontrarse en un seminario en Frankfurt en 1931. Entre ambos se inició un interesante intercambio epistolar en el que un entusiasmado Adorno afirmaba sentir una conmoción intelectual sólo comparable a la de su primer encuentro con Benjamin ${ }^{44}$. Con sus propias modulaciones,

40 Ibid., p. 77.

41 A. Sohn-Rethel. "Gespräch über die Genese der Ideen von Warenform und Denkform”. En: H. Dombrowski (ed.). Symposium Warenform-Denkform. Zur Erkenntnistheorie Sohn-Rethels. Frankfurt a.M.: Campus, 1978, pp. 15-16.

42 Ibid., p. 16.

43 A. Sohn-Rethel. Intellectual and Manual Labour, op. cit., p. XIII.

44 En una carta de 1936 Adorno muestra a Sohn-Rethel su parecer sobre el Exposé: "Querido Alfred, no creo estar exagerando si digo que su carta significó el más importante aval intelectual que yo habría experimentado en la filosofía desde mi primer encuentro con el trabajo de Benjamin, y eso fue en 1923. Este aval muestra la grandeza y la fuerza de su concepción, y también la profundidad de una concordancia entre nuestras ideas que va más allá de lo que usted pueda suponer y de lo que yo mismo podría imaginar. Sólo esta gran concordancia entre nuestras ideas me impide llamar genial a su trabajo, por el recelo de que al final esté refiriéndome también a mi propio trabajo". Th. W. Adorno; A. Sohn-Rethel. Briefwechsel 1936-1969. München: Text+Kritik, 1991, p. 32. 
señalaba el deseo común a su obra y la de Sohn-Rethel de una superación inmanente de la epistemología idealista a partir de una lectura renovada de la filosofía de Marx. Ambos veían en las categorías de Marx los instrumentos adecuados para pensar no sólo los mecanismos que subyacían a la crisis moderna sino también, los que apuntaban a su superación. Por eso, asumieron el motivo marxiano de superar mediante una dialéctica materialista los elementos de determinación ciega bajo los que se encontraban sometidos los sujetos.

Esta admiración ante el trabajo de Sohn-Rethel, sin embargo, no fue compartida por otros miembros del Institut, en especial por Horkheimer, quien vio en sus formulaciones una "excesiva manía de identificación no dialéctica" ${ }^{45}$. Lo cierto es que, pese a su entusiasmo inicial, Adorno tampoco adopta sin fisuras los planteamientos de Sohn-Rethel y se muestra cauteloso ante el peligro de convertir la dialéctica materialista en una prima philosophia que sustituya la hipóstasis del sujeto por una de lo social. Por eso, en la derivación inmediata que hace SohnRethel de la génesis de las categorías desde la abstracción del intercambio detecta ciertos elementos propios de una "doctrina de la copia" que conducen a una peligrosa concepción vulgar del materialismo: "Podría decirse que el concepto es una condición del trabajo social como, también a la inversa, un producto de la abstracción del trabajo social, sin que pueda realmente resolverse la cuestión de qué sea en esa complicada relación la gallina o el huevo" 46 . Frente a toda forma de sustancialización, ya sea la que realiza el idealismo sobre el sujeto como también la propia de un materialismo vulgar sobre la esfera material, la determinación dialéctica que Adorno imprime a su filosofía, y que nos ayuda a diferenciarla de la propuesta de Sohn-Rethel, le lleva a afirmar la necesaria mediación entre ambos. Algo que sólo ve posible en la defensa de una afinidad inmanente entre sujeto y objeto como sedimentos históricos. Esto supondrá, como se verá a continuación, entender el sujeto trascendental kantiano, no sólo como índice de la abstracción idealista, sino también como la representación utópica de la verdadera universalidad social.

\section{Intercambio y "memoria de la totalidad"}

La filosofía de Adorno hizo suyo desde muy pronto el motivo marxiano de superar mediante una dialéctica materialista los elementos de determinación ciega bajo los que se encontraban sometidos los sujetos y que, en última instancia, le impedían alcanzar una organización de la sociedad auténticamente racional. Con Marx, interpretaba la estructura económica de la sociedad burguesa como una de dominio y entendía su deseo de liberar a los hombres de los mecanismos coercitivos que impiden su emancipación como el interés auténtico de la tradición materialista. Por eso, veía en sus categorías los instrumentos adecuados no sólo para pensar los

45 Horkheimer y Adorno tuvieron reacciones opuestas sobre la publicación del texto en la revista del Instituto. Si bien Adorno lo valoró como admirable, Horkheimer encontraba «fatigoso y carente de interés su afirmación constante de que deben presentarse pruebas según las cuales cualquiera de las génesis establecidas a partir del ser (...) coinciden con el problema de la verdad de la consciencia, de la validez del conocimiento o con la praxis de lo social» (Th. W. Adorno; M. Horkheimer. Briefwechsel 1927-1937, vol.1. Frankfurt a.M.: Suhrkamp, 2003, p. 249).

46 Th. W. Adorno. Vorlesung zur Einleitung in die Erkenntnistheorie. Frankfurt a.M.: Junius, 1973, p. 266. 
mecanismos que subyacían a la crisis moderna, sino también aquellos que apuntaban a su superación.

Sin embargo, conservar vivos los elementos de la tradición filosófica ilustrada que veían empezar con Kant y continuar hasta $\operatorname{Marx}^{47}$, no significaba ejercer una mecánica aplicación sobre los problemas del presente, sino que exigía un continuo enriquecimiento de sus conceptos con las nuevas experiencias. Para ello era necesario dejar de tomar la filosofía de Marx como un corpus teórico definitivo y, en su lugar, hacerlo como una teoría cuyos presupuestos exigían ser revisados a fin de reactivar su potencialidad crítica. Acercarse al materialismo marxiano, no como un dogma o catecismo político, sino - en palabras de Benjamin - como una "infraestructura política y filosófica", significaba entenderlo como el diagnóstico de un contexto de relaciones sociales cosificadas y cosificadoras. Pues, como había señalado Horkheimer, su mérito consistió en "haber descubierto la ley del orden inhumano dominante y en mostrar la palanca de la que hay que servirse para crear uno más humano" 48 . Por eso, la lectura actualizada del marxismo debía formular un contenido concreto: una teoría materialista de la sociedad que situara el origen de la miseria y el dolor social en la organización económica burguesa.

Como había hecho Sohn-Rethel, Adorno también asumió la problemática de la teoría marxiana de la mercancía y del dominio social. Ya en su trabajo de habilitación sobre Kierkegaard de 1931 había prestado atención a la categoría de mercancía como "memoria de la totalidad", al tratarse de una categoría en la que se encontraba guardada la conexión completa de todos los fenómenos de la sociedad capitalista. Su desciframiento, aseguraba, permitía explicar el carácter universal de la economía capitalista y, con ello, también la raíz ideológica de la sociedad: "Desplaza de tal forma los acentos de la explicación al lado material, distinto del de la conciencia, que la unidad de una idea de la sociedad capitalista queda destruida por contenidos que no dimanan de ninguna idea, puesto que ponen en cuestión la realidad de la idea misma" ${ }^{49}$. De ella se desprende, pues, la interpretación del intercambio y de la cosificación como factores determinantes en la articulación de su teoría de lo $\operatorname{social}^{50}$. No sólo expresa el estado de parálisis de las instituciones o de los procesos de pensamiento, sino que muestra de qué manera la racionalidad del intercambio en la sociedad capitalista se convierte en el modelo social del principio de identidad. El carácter fetichista de la mercancía no es un hecho de conciencia, sino que también produce conciencia: es la forma de reflexión que tiene ante sí la falsa conciencia. Este es un aspecto central para comprender la especificidad de su lectura del materialismo de Marx frente a la de autores como Lukács, así como la forma en la que reelabora los principios y conceptos fundamentales de su crítica a la economía política ${ }^{51}$. Se trata de la definición de la ley de intercambio como

47 Cfr. H-J. Blank, „Zur Marx-Rezeption des frühen Horkheimer“. En: I. Fetscher Y A. Schmidt (eds.) Emanzipation als Versöhnung. Zu Adornos Kritik der Warentausch-Gesellenschaft und Perspektiven der Transformation. Frankfurt a.M.: Neue Kritik, 2002, p. 53.

48 M. Horkheimer. Dämmerung. En: Gesammelte Schriften, vol. 2. Frankfurt a.M: Fischer, 1998, p. 342.

49 Th. W. Adorno, Kierkegaard. Konstruktion des Ästhetischen. Frankfurt a.M., Suhrkamp, 1962, pp. 151-152.

50 Sobre esto, Zamora insiste en resaltar la manera en la que el principio de intercambio proporciona a Adorno contenidos teóricos-cognoscitivos, que sobrepasan el principio de explicación marxista limitado a la etimología economicista (Cfr. J. A. Zamora, «Lässt sich Kapitalismus immanent kritisieren?”. En: J.M. Romero (ed.) Immanente Kritik heute. Grundlagen und Aktualität eines sozialphilosophischen Begriffs. Bielefeld: Transcript, 2014).

51 Cfr. D. Braunstein. Adornos Kritik der politischen Ökonomie. Bielefeld: transcript, 2011. 
índice de una "mediación universal radical" o "generalidad abstracta" que surge como consecuencia de la subsunción de lo particular en lo general a través de las leyes sociales. Por eso, el intercambio se convierte para Adorno en una categoría histórica-universal que encuentra su origen en el movimiento ilustrado del sujeto frente a la naturaleza:

"La abstracción del principio de intercambio se relaciona con el dominio de lo general sobre lo particular, de la sociedad sobre sus miembros. Por tanto, no es neutral. En la reducción de los individuos a agentes y portadores del intercambio de mercancías se esconde el dominio del hombre sobre el hombre. Eso permanece como algo cierto, pese a todas las dificultades a las que se enfrentan algunas de las categorías de la Crítica a la economía política"52.

Si Marx había atendido a los antagonismos de la sociedad capitalista a partir del estudio de la racionalidad del intercambio, Adorno situó sus análisis en una dialéctica más general que incluyera los momentos no económicos del dominio. Esto significa prestar atención tanto a las antinomias de la racionalidad económica como, sobre todo, a aquellas que se generan en la propia racionalidad dominadora de la naturaleza. "Si tuviera que expresar en una única frase el secreto de la sociedad actual", apunta, "diría que hoy el aparato de reproducción de la vida coincide irremediablemente con el de su dominio" 53 . Con ello pone de relieve la tendencia de la sociedad moderna hacia formas totalitarias en las que los individuos acaban por perder su individualidad al ser sometidos a la totalidad y objetividad social. Esto le permite ver en el intercambio no sólo la causa de la cosificación, sino también la protesta contra la transformación de lo histórico en lo "mítico paralizado". Lo que significa, en última instancia, atender a un impulso de superación inmanente a la propia teoría de la mercancía. Por eso, su crítica no se dirige al principio de intercambio como tal, pretendiendo su definitiva abolición, sino a la abstracción que tiene lugar en él y que desarrolla una forma específica de subjetividad que acaba por sustraer al individuo la autonomía que creía defender. Apunta, pues, al principio de equivalencia e identidad. En esta forma de dominación sin sujeto, en esta máquina que ha prescindido del piloto que ya no necesita, se evidencia la impotencia de los individuos atomizados frente a la lógica niveladora que rige la totalidad social.

La teoría materialista de lo social no debe perder de vista que los individuos no son los sujetos, sino los objetos del proceso social. Esto significa comprender la paradójica mitología burguesa del individuo: lo presenta como una subjetividad fuerte que logra su identidad dominando la objetividad, cuando en realidad los individuos son desgajados y arrojados sin protección a la "ley natural" del intercambio capitalista, convirtiéndolos en seres depotenciados, empobrecidos e incapaces de comprender la realidad en la que se encuentran. El yo-endurecido, capacitado para sobrevivir adaptándose en medio de la administración total, paga el precio de su adaptación con el adiestramiento de su sustrato somático y afectivo.

52 Th. W. Adorno. "Gesellschaft”. En: GS, vol. 8. Frankfurt a.M., Suhrkamp, 1972, p. 13.

53 Th. W. Adorno. "Graeculus II. Notizen zur Philosophie und Gesellschaft 1943-1969". En: Frankfurter Adorno Blätter VIII. München: Text+Kritik, 2003, p. 9. 


\section{El sujeto como forma de reflexión sobre la falsa objetividad}

En Dialéctica de la Ilustración, Adorno y Horkheimer habían prestado atención a la forma en la que el pensamiento kantiano reflejaba una división propia de la filosofía moderna. De un lado, el sujeto trascendental revela la imagen utópica de una libre organización racional de los individuos, que logra superar las diferencias particulares. De otro, también presenta la razón como mecanismo de cálculo y control que organiza el mundo para su autoconservación. El resultado, dirán, es la confirmación de las contradicciones no resueltas que se dan en la "oscura relación del yo trascendental con el yo empírico" 54 . Poner de manifiesto estas aporías y contradicciones se convierte en la tarea fundamental de la metacrítica materialista de Adorno. A partir de la reflexión sobre sus componentes y formas de fundamentación, trata de poner al descubierto la inclusión de un contenido histórico y social concreto en la teoría del conocimiento. Pues en las supuestas categorías formales del pensamiento, ve oculto un contenido social resultado de la imbricación entre naturaleza e historia. Con Sohn-Rethel, insiste en la necesidad de comprender de qué manera la síntesis social permanece escondida en la unidad trascendental de la conciencia tal como aparece en la filosofía del sujeto: "No sólo el yo puro se encuentra mediado por uno empírico sino que también lo está principio trascendental en el cual la filosofía cree poseer su primero frente a lo existente cambiante" 55 .

El principio de identidad que reduce el mundo entero a lo homogéneo e idéntico, tiene su modelo social en el intercambio. En el esquematismo de la razón pura que hace concordar lo universal con el caso singular, no sólo ve una muestra de hybris subjetiva de la teoría moderna del conocimiento, sino también la expresión del dominio que provoca la objetividad social que pesa sobre el sujeto viviente ${ }^{56}$. Lo se encuentra codificado en la teoría del conocimiento kantiana, asegura, es la teodicea de la propia "existencia burguesa". Por eso, insiste en ver en la teoría del conocimiento de Kant las huellas de

"un pensamiento que quiere establecer la validez del momento de lo no-idéntico de la manera más enfática posible. Es un pensamiento que no se agota en que todo sea reducido al pensamiento mismo, sino que se permite criticar la superstición de que todo brota de los seres humanos, a la manera ilustrada, como a cualquier otra superstición, y decir precisamente que la absolutización de aquello que el ser humano sea no es muy distinta a la que sucede con las prácticas de los chamanes, que consideran sus ritos como objetivamente significativos, aunque en realidad sólo se trata de abracadabras subjetivos" ${ }^{97}$.

Si bien Adorno sigue a Sohn-Rethel en este punto, la determinación negativa que imprime a sus tesis de una necesaria lectura dialéctica y mediada de la noción idealista de conciencia trascendental plantea importantes diferencias entre ambos

\footnotetext{
54 Th. W. Adorno; M. Horkheimer. Dialektik der Aufklärung. En: GS, vol. 3. Frankfurt a.M., Suhrkamp, 1970 , p. 102.

55 Th. W. Adorno. Ontologie und Dialektik. Frankfurt a. M: Suhrkamp, 2003, p. 337.

56 Para Bernstein, la generalidad del sujeto trascendental permite ser pensada como "el contexto funcional de una sociedad como un todo que emerge de las espontaneidades, pero las limita y las supera" (J. Bernstein, "Negative Dialektik. Begriff und Kategorien III. Adorno zwischen Kant und Hegel”. En: C. Menke (ed.), Theodor W. Adorno. Negative Dialektik. Berlin: Akademie Verlag, 2006, p. 100).

57 Th. W. Adorno, Kants Kritik, op. cit., p.104.
} 
autores. Y es que para el teórico frankfurtiano, el sujeto trascendental esconde un doble contenido de verdad y no-verdad. De un lado, contiene el momento utópicoilustrado que responde a la idea de universalidad que va más allá de los individuos y de la forma particular de una sociedad en un momento determinado. De otro lado, su universalidad es la de la totalidad social del intercambio, donde la abstracción genera una forma de dominio social como integración de lo particular en lo general. Por eso, paradójicamente, no sólo representa lo más abstracto, sino precisamente por ello también lo más real. Este será el momento de verdad que otorgue a la conciencia trascendental, pues "más allá del círculo mágico de la filosofía de la identidad, el sujeto trascendental representa la sociedad inconsciente de sí misma" 58 .

La especificidad de las tesis materialistas de Adorno frente a las conclusiones de los análisis de Sohn-Rethel se deja ver en la determinación inmanente de su crítica. Pues gana su posición frente al idealismo desde las propias categorías de la filosofía del sujeto. El momento de prioridad del objeto entendido como la "preformación social de toda experiencia individual" 59 , se encuentra contenido desde el principio en los presupuestos de la filosofía del sujeto, de ahí que sólo a partir del análisis inmanente de sus categorías - esto es, en un segundo momento de reflexión en el interior de la filosofía de la conciencia - es posible el ejercicio de una crítica inmanente al sujeto. "No se puede acceder filosóficamente a las categorías sociales sino mediante el desciframiento del concepto de verdad de las categorías filosóficas" $" 60$, señalaba en Dialéctica negativa, por lo que el contenido de verdad oculto en la unidad trascendental de la conciencia exige ser analizado a partir del proceso de cosificación y de racionalización que tiene lugar en la sociedad moderna. "Lo firme, lo persistente e impenetrable del yo es la mímesis de la impenetrabilidad percibida por la conciencia primitiva del mundo exterior" ${ }^{\text {" }}$. Su abstracción y universalidad no son una quimera derivada de una clase de hybris del sujeto, sino la expresión de la esencia social del trabajo, pues tiene su origen en los procesos de abstracción y de dominación de la razón instrumental bajo la forma del principio social del intercambio. Por eso, más allá de sus implicaciones epistemológicas, Adorno encuentra en la teoría kantiana del conocimiento un contenido histórico propio:

«la estructura antinómica del sistema kantiano expresaba, más que contradicciones en que la especulación sobre objetos metafísicos se enreda necesariamente, algo perteneciente a la filosofía de la historia (...) La influencia poderosa de la Crítica de la razón pura ha de atribuirse a la fidelidad con la que consignó el nivel de experiencia de la conciencia» ${ }^{62}$.

Esto no quiere decir, sin embargo, que sea posible identificar sin más sujeto trascendental y sociedad (si bien en uno de sus cursos reconoció la tentación que había tenido en ocasiones), sino poner de manifiesto el hecho de que sólo el sujeto social total (y no el individuo viviente) tiene el carácter de universalidad que Kant atri-

58 Th. W. Adorno. Ontologie und Dialektik, op. cit., p. 337.

59 Th. W. Adorno, Zur Metakritik der Erkenntnistheorie. En: GS, vol. 5, Frankfurt a. M.: Suhrkamp, 1970 , p. 160.

60 Th. W. Adorno, Negative Dialektik, op. cit., p. 198.

61 Ibid., p. 180.

62 Ibid., p. 374. 
buyó a la unidad de la consciencia trascendental: "Aquello que desde la Crítica de la razón pura representa la esencia del sujeto trascendental, la funcionalidad o la actividad pura (...), no sólo es análogo a la teoría del valor, sino que es proyectada a un sujeto puro como su fundamento"63. La síntesis trascendental no sólo refleja las condiciones de la objetividad del conocimiento, sino también la forma de la sociedad como sistema funcional y objetivo. De ahí que Adorno funde su crítica al conocimiento como crítica de la sociedad, y viceversa. La filosofía trascendental muestra la transformación del sistema social en un contexto racional y objetivo, un mundo administrado que subsume lo individual, identificándolo y manteniéndolo en una unidad. La estandarización se convierte entonces en una categoría válida para determinar tanto lo que sucede a las mercancías como a los hombres. Esta es la paradoja que se da en un sistema social que dota a los sujetos de autonomía y libertad para, al mismo tiempo, impedirles ponerlas en práctica. $Y$ es que cuanto mayor sea el grado de sumisión de los individuos a las funciones de la totalidad social, mayor será también la elevación de la categoría de sujeto a principio de dominación. De esta manera, no hay oposición entre subjetivismo y cosificación, sino que entiende la cosificación como una función de aquella, "pues cuanto más cosificación haya, más subjetivización se dará en la filosofía" ${ }^{4}$. Adorno detecta un impulso cosificador en los intentos de referir los fenómenos a la unidad idéntica del sujeto, tal como aparece en la epistemología idealista. El resultado es la polarización del sujeto y el objeto, de manera que cuanto mayor sea la reducción del conocimiento al sujeto, mayor es también la del objeto a mera determinación. El "olvido de la mediación" - esto es, del ser material del sujeto y de la necesaria intervención subjetiva en el objeto - será interpretado por el frankfurtiano como una forma de cosificación. La cosificación es el olvido de la génesis como resultado de la hipóstasis idealista del sujeto, por lo que la tarea de una filosofía materialista negativa será desvelar este olvido como ideológico.

La disolución del mundo en la actividad del sujeto y la cosificación del mundo como algo acabado, terminan por coincidir. Así, el más que proclama el sujeto trascendental, es el menos del sujeto viviente. Por eso, para Adorno, la filosofía moderna no sólo es reductio ad hominem (reducción de la naturaleza y del objeto al espíritu), sino en virtud de sí misma, reductio hominis, reducción del sujeto real, descualificado y reducido. Aquí ve la antinomia de la sociedad burguesa en general:

“que, por un parte, está progresando cada vez más la racionalidad de los seres humanos, puesto que las personas han hecho cada vez más el mundo a su imagen y semejanza, y cada vez más lo han organizado como algo propio. Pero, por otro lado, el mundo se ha convertido en algo que, frente a ellos, les domina, en algo frente a lo que son propiamente heterónomos, en algo con lo que ya no pueden despacharse. Y están enfrentados a él como de manera tan impotente como nosotros estamos hoy enfrentados al mundo, que tiene tal poder no sólo sobre nosotros, sino también sobre nuestro pensamiento, que no somos capaces de enfrentarnos a él" ${ }^{\prime \prime 5}$.

\footnotetext{
63 Th. W. Adorno. Ontologie und Dialektik, op. cit, p. 338.

64 Th. W. Adorno. Kants Kritik, op. cit., p. 174.

65 Th. W. Adorno, Kants Kritik, op. cit., pp. 175-176.
} 


\section{Conclusiones}

En una de sus cartas, Adorno defendía ante Sohn-Rethel que uno de los objetivos más importantes de la dialéctica materialista era "liquidar la idea de la prima philosophia y colocar en su lugar una ultima philosophia"66.

Si Sohn-Rethel atendía a los antagonismos de la sociedad capitalista a partir del estudio de la racionalidad del intercambio, Adorno sitúa sus análisis en una dialéctica más general que le permite ver en éste no sólo la causa de la cosificación, sino también el momento de protesta contra la transformación de lo histórico en segunda naturaleza. Esto significa atender a un impulso de superación inmanente a la propia teoría de la mercancía. Encuentra en lo cosificado no sólo la expresión del sometimiento de los individuos bajo las condiciones dominantes de producción, sino también de la presencia de lo objetivo irreductible al sujeto, la "figura deformada de lo no-idéntico" a la que apunta la utopía materialista bajo el nombre de "prioridad del objeto". Ella alude, por un lado, a la cosificación real que tiene lugar en la sociedad del intercambio. Pero, por otro, también sirve a la dialéctica negativa como momento de protesta y de resistencia crítica ante la negatividad social.

Por eso, la prioridad del objeto exige la adopción de barreras frente a una primacía de la subjetividad abstracta, pero también propone un sujeto real al que le correspondería un objeto no subsumido ni reducido ${ }^{67}$. La crítica inmanente del idealismo sólo es posible a partir de un concepto enfático de subjetividad del que adolece la filosofía de Sohn-Rethel y que lo incapacita para ver el contenido utópico materialista que se encuentra encerrado en el sistema idealista. Para Adorno el sujeto de la experiencia histórica y filosófica es el existente empíricamente, pues el potencial de cualidades de la cosa espera, no el residuo trascendental, sino un sujeto cualitativo. Por eso, la esperanza de la emancipación reside en la capacidad del sujeto para reconocer su dependencia respecto a las condiciones sociales determinantes y encontrar así "el potencial para cancelar y superar su propio señorío". Esta exigencia de "más sujeto" se interpreta entonces como un segundo giro copernicano dentro de la filosofía del sujeto. Y hacia ahí se dirige la utopía materialista adorniana, esto es, hacia la búsqueda de espacios de emergencia para el individuo en la era de su aniquilación ${ }^{68}$.

En su acercamiento a las tesis de Marx y Kant, el materialismo en Adorno aparece como diagnóstico e interpretación crítico de las figuras de la teoría del conocimiento y de los fragmentos de la realidad. Se trata de un pensamiento orientado a la posibilidad de abrir una relación distinta entre el sujeto y el objeto, entre teoría y praxis, entre teoría del conocimiento y análisis social. En ella se expresa el impulso inherente a todo pensamiento materialista por desenmascarar la primacía del momento espiritual y por presentarse ante el sistema idealista como su límite, mostrándole el momento de naturalidad y de objetividad que le es inmanente. Pero también toda tesis materialista incluye un momento de regresión cuando opone a la identificación con el espíritu absoluto la defensa del carácter inmediato de lo material. En esta tensión se mueve la dialéctica negativa. Se dirige contra la primacía abstracta del

66 Th. W. Adorno; A. Sohn-Rethel. Briefwechsel, op. cit., p. 11.

${ }_{67}$ Como señala Thyen, la prioridad del objeto no significa la negación del sujeto, pues «se gana a partir de un modo deficiente del concepto de mediación idealista, y no a través de la negación del sujeto» (A. Thyen. Negative Dialektik und Erfahrung. Zur Rationalität des Nichtidentischen bei Adorno. Frankfurt a.M.: Suhrkamp, 1989, p. 208).

${ }_{68}$ Cfr. R. Tiedemann. Niemandsland. Studien mit und über Th. W. Adorno. München, Text+Kritik, 2007, p. 23 
sujeto y, a la vez, contra la pretensión de una identidad entre verdad y razón. Quiere mostrar el papel del componente material para el conocimiento y, sin embargo, la determinación negativa que imprime sobre sus tesis materialistas le impide caer en una forma de ontología que claudique en lo material inmediato. En este sentido, sus tesis materialistas apuntan a una doble dirección: a la crítica a la falsa subjetividad y a la falsa objetividad. Frente a ambas direcciones es posible leer la orientación abierta y no-dogmática de su materialismo.

\section{Referencias bibliográficas}

Adorno, Th. W. Negative Dialektik, en: Gesammelte Schriften (en adelante, GS), vol. 6. Frankfurt a.M.: Suhrkamp, 1970.

—Zur Metakritik der Erkenntnistheorie, en: GS, vol. 5. Frankfurt a. M., Suhrkamp, 1970.

—-Gesellschaft", en: GS, vol. 8. Frankfurt a.M., Suhrkamp, 1972.

—Vorlesung zur Einleitung in die Erkenntnistheorie. Frankfurt a.M.: Junius, 1973.

—_Zu Subjekt und Objekt”, en: GS, vol. 10.2. Frankfurt a.M., Suhrkamp, 1977.

—Kants Kritik der reinen Vernunft. Frankfurt a.M.: Suhrkamp, 1995.

-Ontologie und Dialektik. Frankfurt a. M: Suhrkamp, 2003.

—Vorlesung über Negative Dialektik. 1965-66. Frankfurt a.M.: Suhrkamp, 2003.

—_Graeculus II. Notizen zur Philosophie und Gesellschaft 1943-1969", en: Frankfurter Adorno Blätter VIII. München: Text+Kritik, 2003.

Adorno, Th. W.; Horkheimer, M. Briefwechsel 1927-1937. Frankfurt a.M.: Suhrkamp, 2003.

Adorno, Th. W.; Krenek. E., Briefwechsel. Frankfurt a.M.: Suhrkamp, 1974.

Adorno, Th. W.; Sohn-Rethel, A. Briefwechsel 1936-1969. München: text+kritik, 1991.

Braun, C. Kritische Theorie vs Kritizismus. Zur Kant-Kritik Theodor W. Adornos. Berlin: de Gruyer, 1983.

Braunstein, J. Adornos Kritik der politischen Ökonomie. Bielefeld: transcript, 2011.

Dombrowski, H. (ed.) Symposium Warenform-Denkform. Zur Erkenntnistheorie Sohn-Rethels. Frankfurt a.M.: Campus, 1978.

Hörisch, J. "Identitätszwang und Tauschabstraktion. Alfred Sohn-Rethels soziogenetische Erkenntnistheorie", en: Geld und Geltung. Zu Alfred Sohn-Rethels soziologischer Erkenntnistheorie. Würzburg: Königshausen \& Neumann, 2006.

Horkheimer, M., "Wissenschaft und Krise zwischen Idealismus und Materialismus", en: Gesammelte Schriften, vol. 12. Frankfurt a.M.: Fischer, 1985.

Kordela, A. "Materialist Epistemology: Sohn-Rethel with Marx and Spinoza". History of the Human Sciences, vol. 29(2), 2016.

Menke, C. (ed.), Theodor W. Adorno. Negative Dialektik. Berlin: Akademie Verlag, 2006.

Ramas San Miguel, C., Fetiche y mistificación capitalistas. La crítica de la economía política de Marx. Tres Cantos: Siglo XXI, 2018.

Sohn-Rethel, A. Intellectual and Manual Labour. New Jersey: Humanities Press, 1978.

Sohn-Rethel, A, Soziologische Theorie der Erkenntnis. Frankfurt a. M.: Suhrkamp, 1985.

Sohn-Rethel, A. Geistige und körperliche Arbeit. Zur Epistemologie der abendländlische Geschichte. Weinheim: VCH, Acta Humaniora, 1989.

Sohn-Rethel, A. Von der Analytik des Wirtschaftens zur Theorie der Volkswirtschaft. Frühe Schriften. Freiburg i.B.: Ça-ira Verlag, 2012.

Thein, C. Subjekt und Synthesis. Eine kritische Studie zum Idealismus und seiner Rezeption bei Adorno, Habermas und Brandom. Würzburg, Königshausen \& Neumann, 2013. 
Thyen, A. Negative Dialektik und Erfahrung. Zur Rationalität des Nichtidentischen bei Adorno. Frankfurt a.M.: Suhrkamp, 1989.

Tiedemann, R. Niemandsland. Studien mit und über Th. W. Adorno. München: text+kritik, 2007.

Woesler, C. Für eine be-greifende Praxis in der Natur. Geldförmige Naturerkenntnis und kybernetische Natur. Giessen: Focus-Verlag, 1978.

Wohlrapp, H. "Materialistische Erkenntniskritik? Kritik an Alfred Sohn-Rethels Ableitung des abstrakten Denkens und Erörterung einiger grundlegender Gesichtspunkte für eine mögliche materialistische Erkenntnistheorie", en: Mittelstraß, J. (ed.) Methodologische Probleme einer normativkritischen Gesellschaftstheorie. Frankfurt a.M.: Suhrkamp, 1975.

Zamora, J.A. «Lässt sich Kapitalismus immanent kritisieren?”, en: Romero, J.M. (ed.) Immanente Kritik heute. Grundlagen und Aktualität eines sozialphilosophischen Begriffs. Bielefeld, Transcript, 2014. 